\title{
A higher-order nonlocal three-point boundary value problem for sequential fractional differential equations
}

\author{
Bashir Ahmad and Sotiris K. Ntouyas
}




\title{
A HIGHER-ORDER NONLOCAL THREE-POINT BOUNDARY VALUE PROBLEM FOR SEQUENTIAL FRACTIONAL DIFFERENTIAL EQUATIONS
}

\author{
BASHIR AHMAD AND SOTIRIS K. NTOUYAS
}

Received 18 September, 2012

\begin{abstract}
This paper investigates the existence of solutions for a sequential fractional differential equation of arbitrary order $\xi+1, \xi \in(n-1, n], n \geq 2$ with three-point nonlocal boundary conditions. We make use of standard tools of fixed point theory to establish our existence results. An illustrative example and some special cases are also discussed.
\end{abstract}

2010 Mathematics Subject Classification: 34A08; 34B15

Keywords: differential equation, fractional order, three-point boundary conditions, existence, fixed point

\section{INTRODUCTION}

Initial and boundary value problems of fractional order have extensively been studied by several researchers in recent years. A variety of results ranging from the theoretical aspects of existence and uniqueness of solutions to the analytic and numerical methods for finding solutions have appeared in the literature. Fractional differential equations appear naturally in a number of fields such as physics, biophysics, blood flow phenomena, aerodynamics, electro-dynamics of complex medium, viscoelasticity, electrical circuits, electron-analytical chemistry, biology, control theory, fitting of experimental data, etc. An excellent account in the study of fractional differential equations can be found in [15, 22-24]. For more details and examples, see $[1-4,6,8-10,12,14,17,21,25-27,30]$ and references therein.

The concept of sequential fractional derivative is given, for example, on page 209 of the monograph [20]. There is a close connection between the sequential fractional derivatives and the non sequential Riemann-Liouville derivatives [28,29]. For some recent work on sequential fractional differential equations, we refer the reader to the papers $[7,11,16]$. In [5], the authors studied a sequential fractional differential equation of order $\alpha+1, \alpha \in(1,2]$ with three-point boundary conditions.

In this paper, motivated by [5], we extend the study of sequential fractional differential equations to an arbitrary order. Precisely, for $\xi \in(n-1, n], n \geq 2$, we consider 
a fractional boundary value problem of arbitrary order $\xi+1$ given by

$$
\begin{aligned}
& { }^{c} D^{\xi}(D+\lambda) x(t)=f(t, x(t)), 0<t<1, \\
& x(0)=0, x^{\prime}(0)=0, x^{\prime \prime}(0)=0, \ldots, x^{(n-1)}(0)=0, \\
& x(1)=\alpha x(\sigma), 0<\sigma<1,
\end{aligned}
$$

where ${ }^{c} D$ is the Caputo fractional derivative, $D$ is the ordinary derivative, $f:[0,1] \times$ $\mathbb{R} \rightarrow \mathbb{R}, \lambda$ is a positive real number and $\alpha$ is a real constant.

In Section 2, we present a basic result that lays the foundation for the sequel. The main results, based on Banach's contraction mapping principle, Krasnoselskii's fixed point theorem and nonlinear alternative of Leray-Schauder type, are presented in Section 3.

\section{BASIC RESULT}

Let us recall some basic definitions of fractional calculus [15, 22].

Definition 1. For $(n-1)$-times absolutely continuous function $g:[0, \infty) \rightarrow \mathbb{R}$, the Caputo derivative of fractional order $q$ is defined as

$$
{ }^{c} D^{q} g(t)=\frac{1}{\Gamma(n-q)} \int_{0}^{t}(t-s)^{n-q-1} g^{(n)}(s) d s, n-1<q<n, n=[q]+1,
$$

where $[q]$ denotes the integer part of the real number $q$.

Definition 2. The Riemann-Liouville fractional integral of order $q$ is defined as

$$
I^{q} g(t)=\frac{1}{\Gamma(q)} \int_{0}^{t} \frac{g(s)}{(t-s)^{1-q}} d s, q>0,
$$

provided the integral exists.

Definition 3. Sequential fractional derivative for a sufficiently smooth function $g(t)$ due to Miller-Ross [20] is defined as

$$
D^{\delta} g(t)=D^{\delta_{1}} D^{\delta_{2}} \ldots D^{\delta_{k}} g(t),
$$

where $\delta=\left(\delta_{1}, \ldots, \delta_{k}\right)$ is a multi-index.

In general, the operator $D^{\delta}$ in (2.1) can either be Riemann-Liouville or Caputo or any other kind of integro-differential operator. For instance,

$$
{ }^{c} D^{q} g(t)=D^{-(n-q)}\left(\frac{d}{d t}\right)^{n} g(t), n-1<q<n,
$$

where $D^{-(n-q)}$ is the fractional integral operator of order $n-q$. Here we emphasize that $D^{-p} f(t)=I^{p} f(t), p=n-q$; for more details, see page 87 [22].

Before presenting an auxiliary lemma, we recall the following result [15]. 
Lemma 1. For $q>0$, the general solution of the fractional differential equation ${ }^{c} D^{q} x(t)=0, n-1<q<n$, is given by

$$
x(t)=c_{0}+c_{1} t+c_{2} t^{2}+\ldots+c_{n-1} t^{n-1},
$$

where $c_{0}, c_{1}, \ldots, c_{n-1}$ are arbitrary real constants.

For the forthcoming analysis, we define

$$
\begin{aligned}
P(t)=P_{o}(t) & =\frac{t^{n-1}}{\lambda}-\frac{(n-1) t^{n-2}}{\lambda^{2}}+\frac{(n-1)(n-2) t^{n-3}}{\lambda^{3}} \\
& -\ldots-\frac{(n-1) ! t}{\lambda^{n-1}}+\frac{(n-1) !}{\lambda^{n}}\left(1-e^{-\lambda t}\right), n \text { is odd }, \\
P(t)=P_{e}(t) & =\frac{t^{n-1}}{\lambda}-\frac{(n-1) t^{n-2}}{\lambda^{2}}+\frac{(n-1)(n-2) t^{n-3}}{\lambda^{3}} \\
& -\ldots+\frac{(n-1) ! t}{\lambda^{n-1}}-\frac{(n-1) !}{\lambda^{n}}\left(1-e^{-\lambda t}\right), n \text { is even. }
\end{aligned}
$$

Furthermore, we assume the non-resonance condition, that is, for $P=P_{o}$ and $P=$ $P_{e}$, we choose $\alpha$ such that

$$
P(1)-\alpha P(\sigma) \neq 0 \text {, for } 0<\sigma<1 .
$$

Lemma 2. Assume that the non-resonance condition (2.4) holds. Then, for $v \in$ $C[0,1]$, the unique solution of the equation

$$
{ }^{c} D^{\xi}(D+\lambda) x(t)=v(t), 0<t<1, n-1<\xi \leq n, n \geq 2,
$$

subject to the boundary conditions (1.2) is given by

$$
\begin{aligned}
x(t) & =\int_{0}^{t} e^{-\lambda(t-s)}\left(\int_{0}^{s} \frac{(s-u)^{\xi-1}}{\Gamma(\xi)} v(u) d u\right) d s \\
& +\frac{P(t)}{P(1)-\alpha P(\sigma)}\left[\alpha \int_{0}^{\sigma} e^{-\lambda(\sigma-s)}\left(\int_{0}^{s} \frac{(s-u)^{\xi-1}}{\Gamma(\xi)} v(u) d u\right) d s\right. \\
& \left.-\int_{0}^{1} e^{-\lambda(1-s)}\left(\int_{0}^{s} \frac{(s-u)^{\xi-1}}{\Gamma(\xi)} v(u) d u\right) d s\right],
\end{aligned}
$$

where $P(t)=P_{o}(t)$ and $P(t)=P_{e}(t)$ are given by (2.2) and (2.3) respectively.

Proof. In view of the well known property: ${ }^{c} D^{\xi} I^{\xi} x(t)=x(t)[16,19]$ and Lemma 1 , observe that any solution of the equation

$$
(D+\lambda) x(t)=c_{0}+c_{1} t+c_{2} t^{2}+\ldots+c_{n-1} t^{n-1}+I^{\xi} v(t)
$$

will satisfy (2.5), where $c_{0}, c_{1}, \ldots, c_{n-1}$ are arbitrary unknown constants. Rewriting (2.7), we have

$$
D\left(e^{\lambda t} x(t)\right)=\left[c_{0}+c_{1} t+c_{2} t^{2}+\ldots+c_{n-1} t^{n-1}+I^{\xi} v(t)\right] e^{\lambda t} .
$$


Integrating from 0 to $t$ and using $x(0)=0, x^{\prime}(0)=0, x^{\prime \prime}(0)=0, \ldots, x^{(n-1)}(0)$ $=0$, we get $c_{0}=0, c_{1}=0, c_{2}=0, c_{n-2}=0$ and

$$
x(t)=c_{n-1} P_{o}(t)+\int_{0}^{t} e^{-\lambda(t-s)}\left(\int_{0}^{s} \frac{(s-u)^{\xi-1}}{\Gamma(\xi)} v(u) d u\right) d s, n \text { is odd },
$$

and

$$
x(t)=\hat{c}_{n-1} P_{e}(t)+\int_{0}^{t} e^{-\lambda(t-s)}\left(\int_{0}^{s} \frac{(s-u)^{\xi-1}}{\Gamma(\xi)} v(u) d u\right) d s, n \text { is even, }
$$

where $P_{\boldsymbol{o}}(t)$ and $P_{\boldsymbol{e}}(t)$ are given by (2.2) and (2.3) respectively. Using the condition $x(1)=\alpha x(\sigma)$ in $(2.8)$, we find that

$$
\begin{aligned}
c_{n-1} & =\frac{1}{P_{o}(1)-\alpha P_{o}(\sigma)}\left[\alpha \int_{0}^{\sigma} e^{-\lambda(\sigma-s)}\left(\int_{0}^{s} \frac{(s-u)^{\xi-1}}{\Gamma(\xi)} v(u) d u\right) d s\right. \\
& \left.-\int_{0}^{1} e^{-\lambda(1-s)}\left(\int_{0}^{s} \frac{(s-u)^{\xi-1}}{\Gamma(\xi)} v(u) d u\right) d s\right]
\end{aligned}
$$

where $P_{o}$ is given by (2.2). Similarly, using the condition $x(1)=\alpha x(\sigma)$ in (2.9), we get

$$
\begin{aligned}
\hat{c}_{n-1} & =\frac{1}{P_{e}(1)-\alpha P_{e}(\sigma)}\left[\alpha \int_{0}^{\sigma} e^{-\lambda(\sigma-s)}\left(\int_{0}^{s} \frac{(s-u)^{\xi-1}}{\Gamma(\xi)} v(u) d u\right) d s\right. \\
& \left.-\int_{0}^{1} e^{-\lambda(1-s)}\left(\int_{0}^{s} \frac{(s-u)^{\xi-1}}{\Gamma(\xi)} v(u) d u\right) d s\right]
\end{aligned}
$$

where $P_{e}$ is given by (2.3). Substituting the values of $c_{n-1}, \hat{c}_{n-1}$ respectively in (2.8) and (2.9), we obtain (2.6). This completes the proof.

\section{EXISTENCE OF SOLUTIONS}

Let $\mathcal{C}=C([0,1], \mathbb{R})$ denotes the Banach space of all continuous functions from $[0,1] \rightarrow \mathbb{R}$ endowed with the sup norm defined by $\|x\|=\sup \{|x(t)|, t \in[0,1]\}<\infty$.

For the sake of convenience, we set

$$
P_{1}=\max _{t \in[0,1]}\left|\frac{P(t)}{P(1)-\alpha P(\sigma)}\right|, \quad \rho=\left|\frac{\left(1+P_{1}\right)\left(1-e^{-\lambda}\right)+P_{1} \alpha \sigma^{\xi}}{\lambda \Gamma(\xi+1)}\right|,
$$

where $P(t)$ is given by (2.2), (2.3).

In view of Lemma 2, we transform problem $(1.1)-(1.2)$ as

$$
x=F(x),
$$


where $F: \ell \rightarrow \ell$ is defined by

$$
\begin{aligned}
(F x)(t) & =\int_{0}^{t} e^{-\lambda(t-s)}\left(\int_{0}^{s} \frac{(s-u)^{\xi-1}}{\Gamma(\xi)} f(u, x(u)) d u\right) d s \\
& +\frac{P(t)}{P(1)-\alpha P(\sigma)}\left[\alpha \int_{0}^{\sigma} e^{-\lambda(\sigma-s)}\left(\int_{0}^{s} \frac{(s-u)^{\xi-1}}{\Gamma(\xi)} f(u, x(u)) d u\right) d s\right. \\
& \left.-\int_{0}^{1} e^{-\lambda(1-s)}\left(\int_{0}^{s} \frac{(s-u)^{\xi-1}}{\Gamma(\xi)} f(u, x(u)) d u\right) d s\right] .
\end{aligned}
$$

Observe that problem (1.1) - (1.2) has solutions if the operator equation (3.2) has fixed points.

Theorem 1. Let $f:[0,1] \times \mathbb{R} \rightarrow \mathbb{R}$ be a jointly continuous function satisfying the condition

$$
|f(t, x)-f(t, y)| \leq L|x-y|, \forall t \in[0,1], x, y \in \mathbb{R},
$$

where $L$ is the Lipschitz constant. Further, the non-resonance condition (2.4) holds. Then the boundary value problem (1.1) - (1.2) has a unique solution if $\rho<1 / L$, where $\rho$ is given by (3.1).

Proof. As a first step, we show that the operator $F$ given by (3.2) maps $\mathcal{C}$ into itself. For that, we set $\sup _{t \in[0,1]}|f(t, 0)|=M<\infty$. Then, for $x \in \mathcal{C}$, we have

$$
\begin{aligned}
& \|(F x)\| \\
& =\sup _{t \in[0,1]} \mid \int_{0}^{t} e^{-\lambda(t-s)}\left(\int_{0}^{s} \frac{(s-u)^{\xi-1}}{\Gamma(\xi)} f(u, x(u)) d u\right) d s \\
& +\frac{P(t)}{P(1)-\alpha P(\sigma)}\left[\alpha \int_{0}^{\sigma} e^{-\lambda(\sigma-s)}\left(\int_{0}^{s} \frac{(s-u)^{\xi-1}}{\Gamma(\xi)} f(u, x(u)) d u\right) d s\right. \\
& \left.+\int_{0}^{1} e^{-\lambda(1-s)}\left(\int_{0}^{s} \frac{(s-u)^{\xi-1}}{\Gamma(\xi)} f(u, x(u)) d u\right) d s\right] \mid \\
& \leq \sup _{t \in[0,1]}\left(\int _ { 0 } ^ { t } e ^ { - \lambda ( t - s ) } \left(\int_{0}^{s} \frac{(s-u)^{\xi-1}}{\Gamma(\xi)}(|f(u, x(u))-f(u, 0)|\right.\right. \\
& +|f(u, 0)|) d u) d s) \\
& +\sup _{t \in[0,1]}\left|\frac{P(t)}{P(1)-\alpha P(\sigma)}\right|\left[\alpha \int _ { 0 } ^ { \sigma } e ^ { - \lambda ( \sigma - s ) } \left(\int_{0}^{s} \frac{(s-u)^{\xi-1}}{\Gamma(\xi)}(\mid f(u, x(u))\right.\right.
\end{aligned}
$$




$$
\begin{aligned}
& -f(u, 0)|+| f(u, 0) \mid) d u) d s \\
& +\int_{0}^{1} e^{-\lambda(1-s)}\left(\int_{0}^{s} \frac{(s-u)^{\xi-1}}{\Gamma(\xi)}(|f(u, x(u))-f(u, 0)|\right. \\
& +|f(u, 0)|) d u) d s] \\
& \leq \sup _{t \in[0,1]}\left(\int_{0}^{t} e^{-\lambda(t-s)}\left(\int_{0}^{s} \frac{(s-u)^{\xi-1}}{\Gamma(\xi)}(L|x(u)|+|f(u, 0)|) d u\right) d s\right) \\
& +\sup _{t \in[0,1]}\left|\frac{P(t)}{P(1)-\alpha P(\sigma)}\right|\left[\alpha \int _ { 0 } ^ { \sigma } e ^ { - \lambda ( \sigma - s ) } \left(\int_{0}^{s} \frac{(s-u)^{\xi-1}}{\Gamma(\xi)}(L|x(u)|\right.\right. \\
& +|f(u, 0)|) d u) d s \\
& \left.+\int_{0}^{1} e^{-\lambda(1-s)}\left(\int_{0}^{s} \frac{(s-u)^{\xi-1}}{\Gamma(\xi)}(L|x(u)|+|f(u, 0)|) d u\right) d s\right] \\
& \leq(L\|x\|+M)\left[\sup _{t \in[0,1]}\left(\int_{0}^{t} e^{-\lambda(t-s)}\left(\int_{0}^{s} \frac{(s-u)^{\xi-1}}{\Gamma(\xi)} d u\right) d s\right)\right. \\
& +\sup _{t \in[0,1]}\left|\frac{P(t)}{P(1)-\alpha P(\sigma)}\right|\left\{\alpha \int_{0}^{\sigma} e^{-\lambda(\sigma-s)}\left(\int_{0}^{s} \frac{(s-u)^{\xi-1}}{\Gamma(\xi)} d u\right) d s\right. \\
& \left.\left.+\int_{0}^{1} e^{-\lambda(1-s)}\left(\int_{0}^{s} \frac{(s-u)^{\xi-1}}{\Gamma(\xi)} d u\right) d s\right\}\right] \\
& \leq(L\|x\|+M)\left(\frac{\left(1+P_{1}\right)\left(1-e^{-\lambda}\right)+P_{1} \alpha \sigma^{\xi}}{\lambda \Gamma(\xi+1)}\right) \\
& =(L\|x\|+M) \rho<\infty .
\end{aligned}
$$

This shows that $F$ maps $\mathcal{C}$ into itself. Now, for $x, y \in \mathcal{C}$ and for each $t \in[0,1]$, we obtain

$$
\begin{aligned}
& \|(F x)-(F y)\|=\sup _{t \in[0,1]}|(F x)(t)-(F y)(t)| \\
& \leq \sup _{t \in[0,1]}\left[\int_{0}^{t} e^{-\lambda(t-s)}\left(\int_{0}^{s} \frac{(s-u)^{\xi-1}}{\Gamma(\xi)}|f(u, x(u))-f(u, y(u))| d u\right) d s\right.
\end{aligned}
$$




$$
\begin{aligned}
& +\left|\frac{P(t)}{P(1)-\alpha P(\sigma)}\right|\left\{\alpha \int _ { 0 } ^ { \sigma } e ^ { - \lambda ( \sigma - s ) } \left(\int_{0}^{s} \frac{(s-u)^{\xi-1}}{\Gamma(\xi)} \mid f(u, x(u))\right.\right. \\
& -f(u, y(u)) \mid d u) d s \\
& \left.\left.+\int_{0}^{1} e^{-\lambda(1-s)}\left(\int_{0}^{s} \frac{(s-u)^{\xi-1}}{\Gamma(\xi)}|f(u, x(u))-f(u, y(u))| d u\right) d s\right\}\right] \\
& \leq L\|x-y\|\left[\sup _{t \in[0,1]}\left(\int_{0}^{t} e^{-\lambda(t-s)}\left(\int_{0}^{s} \frac{(s-u)^{\xi-1}}{\Gamma(\xi)} d u\right) d s\right)\right. \\
& +\sup _{t \in[0,1]}\left|\frac{P(t)}{P(1)-\alpha P(\sigma)}\right|\left\{\alpha \int_{0}^{\sigma} e^{-\lambda(\sigma-s)}\left(\int_{0}^{s} \frac{(s-u)^{\xi-1}}{\Gamma(\xi)} d u\right) d s\right. \\
& \left.\left.+\int_{0}^{1} e^{-\lambda(1-s)}\left(\int_{0}^{s} \frac{(s-u)^{\xi-1}}{\Gamma(\xi)} d u\right) d s\right\}\right] \\
& \leq L\left|\frac{\left(1+P_{1}\right)\left(1-e^{-\lambda}\right)+P_{1} \alpha \sigma^{\xi}}{\lambda \Gamma(\xi+1)}\right|\|x-y\| \\
& =\rho L\|x-y\|,
\end{aligned}
$$

where $\rho$ is given by (3.1). As $\rho<1 / L$, therefore, $F$ is a contraction. Thus, the conclusion of the theorem follows by the contraction mapping principle. This completes the proof.

Now, we state a known result due to Krasnoselskii [18] which is needed to prove the existence of at least one solution of $(1.1)-(1.2)$.

Theorem 2. Let $M$ be a closed, convex, bounded and nonempty subset of a Banach space X. Let $\mathscr{E}_{1}, \mathscr{E}_{2}$ be the operators such that: (i) $\mathscr{E}_{1} x+\mathscr{E}_{2} y \in M$ whenever $x, y \in M$; (ii) $\mathscr{E}_{1}$ is compact and continuous; (iii) $\mathscr{E}_{2}$ is a contraction mapping. Then there exists $z \in M$ such that $z=\mathscr{E}_{1} z+\mathscr{E}_{2} z$.

Theorem 3. Assume that $f:[0,1] \times \mathbb{R} \rightarrow \mathbb{R}$ is a jointly continuous function and the following assumptions hold:

$\left(\mathbf{H}_{\mathbf{1}}\right)|f(t, x)-f(t, y)| \leq L|x-y|, \forall t \in[0,1], x, y \in \mathbb{R}$;

$\left(\mathbf{H}_{2}\right)|f(t, x)| \leq \mu(t), \quad \forall(t, x) \in[0,1] \times \mathbb{R}$ with $\mu \in C([0,1], \mathbb{R})$.

Further, the non-resonance condition (2.4) holds. Then the boundary value problem (1.1)-(1.2) has at least one solution on $[0,1]$ if

$$
\left|\frac{P_{1}\left(1-e^{-\lambda}+\alpha \sigma^{\xi}\right)}{\lambda \Gamma(\xi+1)}\right|<1 .
$$


Proof. Letting $\sup _{t \in[0,1]}|\mu(t)|=\|\mu\|$, we fix

$$
r \geq\left|\frac{\left(1+P_{1}\right)\left(1-e^{-\lambda}\right)+P_{1} \alpha \sigma^{\xi}}{\lambda \Gamma(\xi+1)}\right|\|\mu\|,
$$

and consider $\mathscr{B}_{r}=\{x \in \mathcal{C}:\|x\| \leq r\}$. Define the operators $F_{1}$ and $F_{2}$ on $\mathscr{B}_{r}$ as

$$
\begin{aligned}
\left(F_{1} x\right)(t) & =\int_{0}^{t} e^{-\lambda(t-s)}\left(\int_{0}^{s} \frac{(s-u)^{\xi-1}}{\Gamma(\xi)} f(u, x(u)) d u\right) d s, \\
\left(F_{2} x\right)(t) & =\frac{P(t)}{P(1)-\alpha P(\sigma)}\left[\alpha \int_{0}^{\sigma} e^{-\lambda(\sigma-s)}\left(\int_{0}^{s} \frac{(s-u)^{\xi-1}}{\Gamma(\xi)} f(u, x(u)) d u\right) d s\right. \\
& \left.-\int_{0}^{1} e^{-\lambda(1-s)}\left(\int_{0}^{s} \frac{(s-u)^{\xi-1}}{\Gamma(\xi)} f(u, x(u)) d u\right) d s\right] .
\end{aligned}
$$

For $x, y \in \mathscr{B}_{r}$, it follows from (3.4) that

$$
\left\|F_{1} x+F_{2} y\right\| \leq\left|\frac{\left(1+P_{1}\right)\left(1-e^{-\lambda}\right)+P_{1} \alpha \sigma^{\xi}}{\lambda \Gamma(\xi+1)}\right|\|\mu\| \leq r .
$$

Thus, $F_{1} x+F_{2} y \in \mathscr{B}_{r}$. In view of the condition (3.3), it can easily be shown that $F_{2}$ is a contraction mapping. The continuity of $f$ implies that the operator $F_{1}$ is continuous. Also, $F_{1}$ is uniformly bounded on $\mathscr{B}_{r}$ as

$$
\left\|F_{1} x\right\| \leq \frac{\left|1-e^{-\lambda}\right|\|\mu\|}{\lambda \Gamma(\xi+1)} .
$$

Now we prove the compactness of the operator $F_{1}$. Setting $\Omega=[0,1] \times \mathscr{B}_{r}$, we define $\sup _{(t, x) \in \Omega}|f(t, x)|=M_{r}$, and consequently we have

$$
\begin{aligned}
& \left\|\left(F_{1} x\right)\left(t_{1}\right)-\left(F_{1} x\right)\left(t_{2}\right)\right\| \\
& =\| \int_{0}^{t_{1}} e^{-\lambda\left(t_{1}-s\right)}\left(\int_{0}^{s} \frac{(s-u)^{\xi-1}}{\Gamma(\xi)} f(u, x(u)) d u\right) d s \\
& -\int_{0}^{t_{2}} e^{-\lambda\left(t_{2}-s\right)}\left(\int_{0}^{s} \frac{(s-u)^{\xi-1}}{\Gamma(\xi)} f(u, x(u)) d u\right) d s \| \\
& \leq \frac{M_{r}}{\lambda \Gamma(\xi+1)}\left(\left|t_{1}^{\xi}-t_{2}^{\xi}\right|+\left|t_{1}^{\xi} e^{-\lambda t_{1}}-t_{2}^{\xi} e^{-\lambda t_{2}}\right|\right),
\end{aligned}
$$

which is independent of $x$ and tends to zero as $t_{2} \rightarrow t_{1}$. Thus, $F_{1}$ is relatively compact on $\mathscr{B}_{r}$. Hence, by the Arzelá-Ascoli Theorem, $F_{1}$ is compact on $\mathscr{B}_{r}$. Thus all the assumptions of Theorem 2 are satisfied and the conclusion of Theorem 2 implies 
that the boundary value problem $(1.1)-(1.2)$ has at least one solution on $[0,1]$. This completes the proof.

In the next theorem we prove the existence of a solution for the boundary value problem (1.1) - (1.2) via Leray-Schauder nonlinear alternative.

Lemma 3 (Nonlinear alternative for single valued maps [13]). Let $E$ be a Banach space, $C$ a closed, convex subset of $E, U$ an open subset of $C$ and $0 \in U$. Suppose that $F: \bar{U} \rightarrow C$ is a continuous, compact (that is, $F(\bar{U})$ is a relatively compact subset of $C$ ) map. Then either

(i) $F$ has a fixed point in $\bar{U}$, or

(ii) there is $a u \in \partial U$ (the boundary of $U$ in $C$ ) and $\lambda \in(0,1)$ with $u=\lambda F(u)$.

Theorem 4. Suppose that $f:[0,1] \times \mathbb{R} \rightarrow \mathbb{R}$ is a jointly continuous function and the non-resonance condition (2.4) is satisfied. Further, it is assumed that the following conditions hold:

$\left(\mathbf{H}_{3}\right)$ There exist a function $\phi \in L^{1}\left([0,1], \mathbb{R}^{+}\right)$, and a nondecreasing function $\psi$ : $\mathbb{R}^{+} \rightarrow \mathbb{R}^{+}$such that $|f(t, x)| \leq \phi(t) \psi(\|x\|)$, for all $(t, x) \in[0,1] \times \mathbb{R}$.

(H) There exists a constant $M>0$ such that

$$
\frac{\|x\|}{\frac{\psi(\|x\|)}{\Gamma(\xi)}\left\{\left(1+P_{1}\right) \int_{0}^{1} e^{-\lambda(1-s)} \phi(s) d s+\alpha P_{1} \int_{0}^{\sigma} e^{-\lambda(\sigma-s)} \phi(s) d s\right\}}>1 .
$$

Then the boundary value problem (1.1)-(1.2) has at least one solution on $[0,1]$.

Proof. Consider the operator $F: \mathcal{\ell} \rightarrow \ell$ where

$$
\begin{aligned}
(F x)(t) & =\int_{0}^{t} e^{-\lambda(t-s)}\left(\int_{0}^{s} \frac{(s-u)^{\xi-1}}{\Gamma(\xi)} f(u, x(u)) d u\right) d s \\
& +\frac{P(t)}{P(1)-\alpha P(\sigma)}\left[\alpha \int_{0}^{\sigma} e^{-\lambda(\sigma-s)}\left(\int_{0}^{s} \frac{(s-u)^{\xi-1}}{\Gamma(\xi)} f(u, x(u)) d u\right) d s\right. \\
& \left.-\int_{0}^{1} e^{-\lambda(1-s)}\left(\int_{0}^{s} \frac{(s-u)^{\xi-1}}{\Gamma(\xi)} f(u, x(u)) d u\right) d s\right] .
\end{aligned}
$$

We show that $F$ maps bounded sets into bounded sets in $C([0,1], \mathbb{R})$. For a positive number $r$, let $\mathscr{B}_{r}=\{x \in C([0,1], \mathbb{R}):\|x\| \leq r\}$ be a bounded set in $C([0,1], \mathbb{R})$. Then

$$
\begin{aligned}
& |(F x)(t)| \\
& \leq \mid \int_{0}^{t} e^{-\lambda(t-s)}\left(\int_{0}^{s} \frac{(s-u)^{\xi-1}}{\Gamma(\xi)} f(u, x(u)) d u\right) d s
\end{aligned}
$$




$$
\begin{aligned}
& +\frac{P(t)}{P(1)-\alpha P(\sigma)}\left[\alpha \int_{0}^{\sigma} e^{-\lambda(\sigma-s)}\left(\int_{0}^{s} \frac{(s-u)^{\xi-1}}{\Gamma(\xi)} f(u, x(u)) d u\right) d s\right. \\
& \left.+\int_{0}^{1} e^{-\lambda(1-s)}\left(\int_{0}^{s} \frac{(s-u)^{\xi-1}}{\Gamma(\xi)} f(u, x(u)) d u\right) d s\right] \mid \\
& \leq \int_{0}^{t} e^{-\lambda(t-s)}\left(\int_{0}^{s} \frac{(s-u)^{\xi-1}}{\Gamma(\xi)} \phi(s) \psi(\|x\|) d u\right) d s \\
& +\left|\frac{P(t)}{P(1)-\alpha P(\sigma)}\right|\left[\alpha \int_{0}^{\sigma} e^{-\lambda(\sigma-s)}\left(\int_{0}^{s} \frac{(s-u)^{\xi-1}}{\Gamma(\xi)} \phi(s) \psi(\|x\|) d u\right) d s\right. \\
& \left.+\int_{0}^{1} e^{-\lambda(1-s)}\left(\int_{0}^{s} \frac{(s-u)^{\xi-1}}{\Gamma(\xi)} \phi(s) \psi(\|x\|) d u\right) d s\right] \\
& \leq \frac{\psi(\|x\|)}{\Gamma(\xi)}\left\{\int_{0}^{1} e^{-\lambda(1-s)} \phi(s) d s+\alpha P_{1} \int_{0}^{\sigma} e^{-\lambda(\sigma-s)} \phi(s) d s\right. \\
& \left.+P_{1} \int_{0}^{1} e^{-\lambda(1-s)} \phi(s) d s\right\} \\
& =\frac{\psi(\|x\|)}{\Gamma(\xi)}\left\{\left(1+P_{1}\right) \int_{0}^{1} e^{-\lambda(1-s)} \phi(s) d s+\alpha P_{1} \int_{0}^{\sigma} e^{-\lambda(\sigma-s)} \phi(s) d s\right\}
\end{aligned}
$$

Consequently,

$$
\|F x\| \leq \frac{\psi(r)}{\Gamma(\xi)}\left\{\left(1+P_{1}\right) \int_{0}^{1} e^{-\lambda(1-s)} p(s) d s+\alpha P_{1} \int_{0}^{\sigma} e^{-\lambda(\sigma-s)} p(s) d s\right\} .
$$

Next we show that $F$ maps bounded sets into equicontinuous sets of $C([0,1], \mathbb{R})$. Let $t_{1}, t_{2} \in[0,1]$ with $t_{1}<t_{2}$ and $x \in \mathscr{B}_{r}$, where $\mathscr{B}_{r}$ is a bounded set of $C([0,1], \mathbb{R})$. Then we obtain

$$
\begin{aligned}
& \left|(F x)\left(t_{2}\right)-(F x)\left(t_{1}\right)\right| \\
& =\mid \int_{0}^{t_{1}}\left(e^{-\lambda\left(t_{2}-s\right)}-e^{-\lambda\left(t_{1}-s\right)}\right)\left(\int_{0}^{s} \frac{(s-u)^{\xi-1}}{\Gamma(\xi)} f(u, x(u)) d u\right) d s \\
& +\int_{t_{1}}^{t_{2}} e^{-\lambda\left(t_{2}-s\right)}\left(\int_{0}^{s} \frac{(s-u)^{\xi-1}}{\Gamma(\xi)} f(u, x(u)) d u\right) d s \\
& +\frac{\left(P\left(t_{2}\right)-P\left(t_{1}\right)\right)}{P(1)-\alpha P(\sigma)}\left[\alpha \int_{0}^{\sigma} e^{-\lambda(\sigma-s)}\left(\int_{0}^{s} \frac{(s-u)^{\xi-1}}{\Gamma(\xi)} f(u, x(u)) d u\right) d s\right.
\end{aligned}
$$




$$
\begin{aligned}
& \left.-\int_{0}^{1} e^{-\lambda(1-s)}\left(\int_{0}^{s} \frac{(s-u)^{\xi-1}}{\Gamma(\xi)} f(u, x(u)) d u\right) d s\right] \mid \\
& \leq\left|\int_{0}^{t_{1}}\left(e^{-\lambda\left(t_{2}-s\right)}-e^{-\lambda\left(t_{1}-s\right)}\right)\left(\int_{0}^{s} \frac{(s-u)^{\xi-1}}{\Gamma(\xi)} \psi(r) \phi(u) d u\right) d s\right| \\
& +\left|\int_{t_{1}}^{t_{2}} e^{-\lambda\left(t_{2}-s\right)}\left(\int_{0}^{s} \frac{(s-u)^{\xi-1}}{\Gamma(\xi)} \psi(r) \phi(u) d u\right) d s\right| \\
& +\mid \frac{\left(P\left(t_{2}\right)-P\left(t_{1}\right)\right)}{P(1)-\alpha P(\sigma)}\left[\alpha \int_{0}^{\sigma} e^{-\lambda(\sigma-s)}\left(\int_{0}^{s} \frac{(s-u)^{\xi-1}}{\Gamma(\xi)} \psi(r) \phi(u) d u\right) d s\right. \\
& \left.-\int_{0}^{1} e^{-\lambda(1-s)}\left(\int_{0}^{s} \frac{(s-u)^{\xi-1}}{\Gamma(\xi)} \psi(r) \phi(u) d u\right) d s\right] \mid
\end{aligned}
$$

Obviously the right hand side of the above inequality tends to zero independently of $x \in \mathcal{B}_{r}$ as $t_{2}-t_{1} \rightarrow 0$. As $F$ satisfies the above assumptions, therefore it follows by the Arzelá-Ascoli theorem that $F: C([0,1], \mathbb{R}) \rightarrow C([0,1], \mathbb{R})$ is completely continuous.

The result will follow from the Leray-Schauder nonlinear alternative (Lemma 3) once we have proved the boundendness of the set of all solutions to equations $x=$ $\lambda F x$ for $\lambda \in[0,1]$.

Let $x$ be a solution. Then, for $t \in[0,1]$, and using the computations in proving that $F$ is bounded, we have

$$
\begin{aligned}
|x(t)| & =|\lambda(F x)(t)| \\
& \leq \frac{\psi(\|x\|)}{\Gamma(\xi)}\left\{\left(1+P_{1}\right) \int_{0}^{1} e^{-\lambda(1-s)} p(s) d s+\alpha P_{1} \int_{0}^{\sigma} e^{-\lambda(\sigma-s)} p(s) d s\right\} .
\end{aligned}
$$

Consequently, we have

$$
\frac{\|x\|}{\frac{\psi(\|x\|)}{\Gamma(\xi)}\left\{\left(1+P_{1}\right) \int_{0}^{1} e^{-\lambda(1-s)} p(s) d s+\alpha P_{1} \int_{0}^{\sigma} e^{-\lambda(\sigma-s)} p(s) d s\right\}} \leq 1 .
$$

In view of $\left(\mathbf{H}_{\mathbf{4}}\right)$, there exists $M$ such that $\|x\| \neq M$. Let us set

$$
U=\{x \in C([0,1], \mathbb{R}):\|x\|<M\} .
$$

Note that the operator $F: \bar{U} \rightarrow C([0,1], \mathbb{R})$ is continuous and completely continuous. From the choice of $U$, there is no $x \in \partial U$ such that $x=\lambda F(x)$ for some $\lambda \in(0,1)$. Consequently, by the nonlinear alternative of Leray-Schauder type (Lemma 3), we deduce that $F$ has a fixed point $x \in \bar{U}$ which is a solution of the problem (1.1)-(1.2). This completes the proof. 
Example 1. Consider the problem

$$
\left\{\begin{array}{c}
{ }^{c} D^{7 / 2}(D+2) x(t)=\frac{L}{2}\left(t^{2}+\cos t+1+x(t)+\tan ^{-1} x(t)\right), 0 \leq t \leq 1, \\
x(0)=0, x^{\prime}(0)=0, x^{\prime \prime}(0)=0, x^{\prime \prime \prime}(0)=0, x(1)=x(1 / 2) .
\end{array}\right.
$$

Here, $\xi=7 / 2, n=4, f(t, x(t))=\frac{L}{2}\left(t^{2}+\cos t+1+x(t)+\tan ^{-1} x(t)\right), \lambda=$ $2, \alpha=1, \sigma=1 / 2$. Clearly

$$
|f(t, x)-f(t, y)| \leq L\left|x-y+\tan ^{-1} x-\tan ^{-1} y\right| / 2 \leq L|x-y|,
$$

$P(t)=P_{e}(t)=t^{3} / 2-3 t(t-1) / 4-3\left(1-e^{-2 t}\right) / 8, \quad P_{1} \approx 6.214821, \rho=[(1+$ $\left.\left.P_{1}\right)\left(1-e^{-2}\right)+2^{-7 / 2} P_{1}\right] / 2 \Gamma(9 / 2) \approx 0.2917760234$.

For $L<1 / \rho \approx 3.42728641$, it follows by Theorem 1 that problem (3.5) has a unique solution.

\section{CONCLUSiOns}

In this paper, we have obtained some existence results for a nonlocal three-point boundary value problem of sequential fractional differential equations of arbitrary order $\xi+1, \xi \in(n-1, n], n \geq 2$. Our results are new and generalize some earlier results. For instance, by taking $n=2$, Theorems 3.1 and 3.3 correspond to Theorems 4.1 and 4.3 of [5]. Letting $\xi=n$, we get the new existence results for the following nonlinear problem of ordinary differential equations:

$$
\begin{gathered}
D^{n}(D+\lambda) x(t)=f(t, x(t)), 0<t<1, \\
x(0)=0, x^{\prime}(0)=0, x^{\prime \prime}(0)=0, \ldots, x^{(n-1)}(0)=0, \\
x(1)=\alpha x(\sigma), 0<\sigma<1 .
\end{gathered}
$$

\section{ACKNOWLEDGEMENT}

The authors thank the reviewers for their useful and constructive comments.

\section{REFERENCES}

[1] B. Ahmad, "Existence of solutions for irregular boundary value problems of nonlinear fractional differential equations," Appl. Math. Lett., vol. 23, no. 4, pp. 390-394, 2010.

[2] B. Ahmad and R. P. Agarwal, "On nonlocal fractional boundary value problems," Dyn. Contin. Discrete Impuls. Syst., Ser. A, Math. Anal., vol. 18, no. 4, pp. 535-544, 2011.

[3] B. Ahmad and J. J. Nieto, "Existence of solutions for nonlocal boundary value problems of higherorder nonlinear fractional differential equations," Abstr. Appl. Anal., vol. 2009, p. 9, 2009.

[4] B. Ahmad and J. J. Nieto, "Existence results for a coupled system of nonlinear fractional differential equations with three-point boundary conditions," Comput. Math. Appl., vol. 58, no. 9, pp. 1838-1843, 2009.

[5] B. Ahmad and J. J. Nieto, "Sequential fractional differential equations with three-point boundary conditions," Comput. Math. Appl., vol. 64, no. 10, pp. 3046-3052, 2012. 
[6] B. Ahmad, J. J. Nieto, and J. Pimentel, "Some boundary value problems of fractional differential equations and inclusions," Comput. Math. Appl., vol. 62, no. 3, pp. 1238-1250, 2011.

[7] C. Bai, "Impulsive periodic boundary value problems for fractional differential equation involving Riemann-Liouville sequential fractional derivative," J. Math. Anal. Appl., vol. 384, no. 2, pp. $211-$ $231,2011$.

[8] Z. Bai, "On positive solutions of a nonlocal fractional boundary value problem," Nonlinear Anal., Theory Methods Appl., Ser. A, Theory Methods, vol. 72, no. 2, pp. 916-924, 2010.

[9] S. Bhalekar, V. Daftardar-Gejji, D. Baleanu, and R. Magin, "Fractional Bloch equation with delay," Comput. Math. Appl., vol. 61, no. 5, pp. 1355-1365, 2011.

[10] D. Băleanu, O. G. Mustafa, and R. P. Agarwal, "An existence result for a superlinear fractional differential equation,” Appl. Math. Lett., vol. 23, no. 9, pp. 1129-1132, 2010.

[11] D. Băleanu, O. G. Mustafa, and R. P. Agarwal, "On $L^{p}$-solutions for a class of sequential fractional differential equations,” Appl. Math. Comput., vol. 218, no. 5, pp. 2074-2081, 2011.

[12] M. Dalla Riva and S. Yakubovich, "On a Riemann-Liouville fractional analog of the Laplace operator with positive energy," Integral Transforms Spec. Funct., vol. 23, no. 4, pp. 277-295, 2012.

[13] A. Granas and J. Dugundji, Fixed point theory, ser. Springer Monographs in Mathematics. New York, NY: Springer, 2003.

[14] V. Keyantuo and C. Lizama, "A characterization of periodic solutions for time-fractional differential equations in UMD spaces and applications," Math. Nachr., vol. 284, no. 4, pp. 494-506, 2011.

[15] A. A. Kilbas, H. M. Srivastava, and J. J. Trujillo, Theory and applications of fractional differential equations, ser. North-Holland Mathematics Studies. Amsterdam: Elsevier, 2006, vol. 204.

[16] M. Klimek, "Sequential fractional differential equations with Hadamard derivative," Commun. Nonlinear Sci. Numer. Simul., vol. 16, no. 12, pp. 4689-4697, 2011.

[17] S. Konjik, L. Oparnica, and D. Zorica, "Waves in viscoelastic media described by a linear fractional model," Integral Transforms Spec. Funct., vol. 22, no. 4-5, pp. 283-291, 2011.

[18] M. Krasnoselskii, "Two remarks on the method of successive approximations," Uspekhi Mat. Nauk., vol. 10, pp. 123-127, 1955.

[19] C. Li and W. Deng, "Remarks on fractional derivatives," Appl. Math. Comput., vol. 187, no. 2, pp. 777-784, 2007.

[20] K. S. Miller and B. Ross, An introduction to the fractional calculus and fractional differential equations. New York: John Wiley \& Sons, Inc., 1993.

[21] J. J. Nieto, "Maximum principles for fractional differential equations derived from Mittag-Leffler functions," Appl. Math. Lett., vol. 23, no. 10, pp. 1248-1251, 2010.

[22] I. Podlubny, Fractional differential equations. An introduction to fractional derivatives, fractional differential equations, to methods of their solution and some of their applications, ser. Mathematics in Science and Engineering. San Diego, CA: Academic Press, 1999, vol. 198.

[23] J. Sabatier, O. P. Agrawal, and J. A. Tenreiro Machado, Eds., Advances in fractional calculus. Theoretical developments and applications in physics and engineering. Dordrecht: Springer, 2007.

[24] S. Samko, A. Kilbas, and O. Marichev, Fractional integrals and derivatives: theory and applications. Transl. from the Russian. New York, NY: Gordon and Breach, 1993.

[25] P. Sztonyk, "Regularity of harmonic functions for anisotropic fractional Laplacians," Math. Nachr., vol. 283, no. 2, pp. 289-311, 2010.

[26] Živorad Tomovski, R. Hilfer, and H. Srivastava, "Fractional and operational calculus with generalized fractional derivative operators and Mittag-Leffler type functions," Integral Transforms Spec. Funct., vol. 21, no. 11-12, pp. 797-814, 2010. 
[27] G. Wang, B. Ahmad, and L. Zhang, "Impulsive anti-periodic boundary value problem for nonlinear differential equations of fractional order," Nonlinear Anal., Theory Methods Appl., Ser. A, Theory Methods, vol. 74, no. 3, pp. 792-804, 2011.

[28] Z. Wei and W. Dong, "Periodic boundary value problems for Riemann-Liouville sequential fractional differential equations," Electron. J. Qual. Theory Differ. Equ., vol. 87, pp. 1-13, 2011.

[29] Z. Wei, Q. Li, and J. Che, "Initial value problems for fractional differential equations involving Riemann-Liouville sequential fractional derivative," J. Math. Anal. Appl., vol. 367, no. 1, pp. 260272, 2010.

[30] S. Zhang, "Positive solutions to singular boundary value problem for nonlinear fractional differential equation," Comput. Math. Appl., vol. 59, no. 3, pp. 1300-1309, 2010.

Authors' addresses

Bashir Ahmad

Department of Mathematics, Faculty of Science, King Abdulaziz University, P.O. Box 80203, Jeddah 21589, Saudi Arabia

E-mail address: bashirahmad-qau@yahoo.com

Sotiris K. Ntouyas

Department of Mathematics, University of Ioannina, 45110 Ioannina, Greece

E-mail address: sntouyas@uoi.gr 